\title{
E-CUSP scheme for the equations of ideal magnetohydrodynamics with high order WENO Scheme
}

\author{
Yiqing Shen ${ }^{\mathrm{a}}$, Gecheng Zha ${ }^{\mathrm{b}}$, Manuel A. Huerta ${ }^{\mathrm{c}, *}$ \\ ${ }^{\mathrm{a}}$ LHD, Institute of Mechanics, Chinese Academy of Sciences, 100190 Beijing, China \\ ${ }^{\mathrm{b}}$ Dept. of Mechanical and Aerospace Engineering, University of Miami Coral Gables, FL 33124, United States \\ ${ }^{\mathrm{c}}$ Dept. of Physics, University of Miami Coral Gables, FL 33124, United States
}

\section{A R T I C L E I N F O}

\section{Article history:}

Available online 25 June 2012

\section{Keywords:}

Hypersonic

MHD

Shocks

\begin{abstract}
A B S T R A C T
An E-CUSP (energy-convective upwind and split pressure) scheme is developed to solve the equations of magnetohydrodynamics. Fifth order WENO reconstructions are employed to calculate the fluxes in order to achieve high order spacial accuracy. A characteristic speed of sound by averaging the fast wave speed and the acoustic speed of sound is suggested to evaluate the Mach number, which will yield robust and accurate solutions. The numerical experiments have demonstrated the accuracy and the capability of the new scheme to capture complex interactions of multiple shocks and vortices.
\end{abstract}

(c) 2012 Elsevier Inc. All rights reserved.

\section{Introduction}

The governing equations of ideal (neglecting the displacement current and electric force) magnetohydrodynamics (MHDs) combine the Euler equations with the pre-Maxwell equations for a fluid with infinite electrical conductivity. However, the electromagnetic field makes the structure of MHD equations more complex than the hydrodynamics equations. There are seven distinct and, in general, non-degenerate eigenvalues in the system of MHD equations. Hence there are seven different waves. In addition to the entropy wave, which propagates at the fluid speed, there are three other wave modes. According to the magnitude of the wave speeds, these three modes are termed as fast, intermediate (Alfven), and slow waves. The fast and slow waves are compressive, while the intermediate wave is not. Depending on the direction and the magnitude of the magnetic field, these wave speeds may coincide. Thus the MHD equations form a non-strictly hyperbolic system [1-5].

Since the ideal MHD equations have a wave-like structure analogous to that of the hydrodynamics equations, various numerical schemes for hydrodynamics equations have been extended to solve the MHD equations in the past two decades. The approximate Riemann solvers, which are based on eigenvalue and eigenvector analysis, are widely used for high speed flows as well as for high speed MHD applications. Beginning with the work of Brio and Wu [1], the numerical methods for MHD equations based on approximate Riemann solvers have extensively been studied and developed. For example, Roe's Riemann solvers are developed by Brio and Wu [1], Dai and Woodward [6], Zachary and Collelaz [7], Roe and Balsara [2] and Cargo and Gallice [8]. HLL (Harten-Lax-van Leer)-type schemes are developed by Janhunen [9], Honkkila and Janhunen [10], Gurski [11], Li [12], Miyoshi and Kusano [13] and Balsara et al. [14]. Flux vector splitting methods are developed by MacCormack [15] and Jiang and Wu [4]. The equations of magnetohydrodynamics are not homogeneous of degree one with respect to the state vector and hence cannot directly perform flux vector splitting. To overcome this difficulty, MacCormack introduces an extra variable $\tilde{a}$ in Refs. [15,16] and adopts the method of Lombard et al. [17]. The flux splitting schemes based

\footnotetext{
* Corresponding author. Tel.: +1 305284 2323; fax: +1 3052844222.

E-mail addresses: yqshen@imech.ac.cn (Y. Shen), gzha@miami.edu (G. Zha), huerta@physics.miami.edu (M.A. Huerta).
} 
on eigenvalues and eigenvectors system are generally very complicated. In our study we use the E-CUSP scheme discussed in Section 2.2, that does not need to use the eigenvector approach.

The low dissipative high order filter schemes developed by Yee and Sjogreen [18] for MHD systems involves a dissipative portion of higher order Lax-Friedrichs scheme or an approximate Riemann solver. Moreover, Balbas [19] developed a central differencing scheme based on the evolution of cell averages over staggered grids. Gaitonde [20] developed a compact difference method for MHD with a local filter switching procedure to change the higher order filter to a second order filter locally for shock capturing. The central differencing scheme and the compact difference scheme do not need a detailed knowledge of the eigenstructure of the Jacobian matrices. However, the central differencing schemes have difficulty in capturing shock waves in monotonic fashion.

In recent years, the convective upwind and split pressure (CUSP) family schemes, which simultaneously consider the convective upwind characteristics and avoid the complex eigen-decomposition process, have achieved great success in gasdynamics. The CUSP schemes can be basically categorized to two types, the H-CUSP and E-CUSP [21-23]. The H-CUSP schemes have the total enthalpy from the energy equation in their convective vector, whereas the E-CUSP schemes use the total energy in the convective vector. The Liou's AUSM family schemes [24-28], Van Leer-Hänel scheme [29], and Edwards's LDFSS schemes [30,31] belong to the H-CUSP group. The schemes developed by Zha et al. [32-36] belong to the E-CUSP group.

Most of the CUSP schemes mentioned above are low diffusive. However, as discussed in [37], the low diffusion scheme combined with high-order reconstruction is more prone to yield numerical oscillations in a shock wave. Agarwal et al. [38] applied the original AUSM method with first-order spatial accuracy to one-dimensional MHD cases. Han et al. [37] developed a AUSMPW+/M-AUSMPW + schemes combined with the MLP (multidimensional limiting process) interpolation method to achieve the higher order accuracy for MHD equations.

In this paper, an E-CUSP scheme based on the one suggested by Zha et al. [36] is developed for MHD system. This scheme avoids the complication of deriving the eigenvalues and eigenvector system when the MHD equations are incorporated. The new E-CUSP scheme is used with a high order WENO reconstruction for the magnetohydrodynamics equations. The numerical experiments demonstrate the new scheme's accuracy and robustness.

\section{Numerical method}

\subsection{Governing equations}

The ideal MHD equations for inviscid flow can be expressed in vector form as [39]

$$
\frac{\partial \mathbf{U}}{\partial t}+\nabla \cdot \mathbf{F}=0
$$

where

$$
\begin{aligned}
& \mathbf{U}=\left(\begin{array}{l}
\rho \\
\rho \mathbf{V} \\
\mathbf{B} \\
\rho e
\end{array}\right), \quad \mathbf{F}=\left(\begin{array}{l}
\rho \mathbf{V} \\
\rho \mathbf{V} \mathbf{V}+p_{t} \mathbf{I}-\mathbf{B B} \\
\mathbf{V B}-\mathbf{B V} \\
\left(\rho e+p_{t}\right) \mathbf{V}-\mathbf{B}(V \cdot B)
\end{array}\right), \\
& p_{t}=p+\frac{1}{2} B^{2}, \quad \rho e=\frac{1}{2} \rho \mathbf{V}^{2}+\frac{1}{2} \mathbf{B}^{2}+\frac{p}{(\gamma-1)},
\end{aligned}
$$

Subject to the constraint

$$
\nabla \cdot \mathbf{B}=0
$$

where $\rho$ is the flow density, $\mathbf{V}$ is the velocity vector, $\rho$ e is the energy, $p$ is the pressure, $\mathbf{B}$ is the magnetic field.

The governing equations. (Eq. (1)) can be written in the Cartesian coordinate as:

$$
\frac{\partial \mathbf{U}}{\partial t}+\frac{\partial \mathbf{E}}{\partial x}+\frac{\partial \mathbf{F}}{\partial y}+\frac{\partial \mathbf{G}}{\partial z}=0,
$$

where

$$
\mathbf{U}=\left[\begin{array}{l}
\rho \\
\rho u \\
\rho v \\
\rho w \\
\rho e \\
B_{x} \\
B_{y} \\
B_{z}
\end{array}\right], \quad \mathbf{E}=\left[\begin{array}{l}
\rho u \\
\rho u^{2}+p_{t}-B_{x}^{2} \\
\rho u v-B_{x} B_{y} \\
\rho u w-B_{x} B_{z} \\
\left(\rho e+p_{t}\right) u-B_{x}\left(u B_{x}+v B_{y}+w B_{z}\right) \\
u B_{x}-u B_{x} \\
u B_{y}-v B_{x} \\
u B_{z}-w B_{x}
\end{array}\right]
$$




$$
\mathbf{F}=\left[\begin{array}{l}
\rho v \\
\rho u v-B_{y} B_{x} \\
\rho v^{2}+p_{t}-B_{y}^{2} \\
\rho v w-B_{y} B_{z} \\
\left(\rho e+p_{t}\right) v-B_{y}\left(u B_{x}+v B_{y}+w B_{z}\right) \\
v B_{x}-u B_{y} \\
v B_{y}-v B_{y} \\
v B_{z}-w B_{y}
\end{array}\right], \quad \mathbf{G}=\left[\begin{array}{l}
\rho w \\
\rho u w-B_{z} B_{x} \\
\rho v w-B_{z} B_{y} \\
\rho w^{2}+p_{t}-B_{z}^{2} \\
\left(\rho e+p_{t}\right) w-B_{z}\left(u B_{x}+v B_{y}+w B_{z}\right) \\
w B_{x}-u B_{z} \\
w B_{y}-v B_{z} \\
w B_{z}-w B_{z}
\end{array}\right] .
$$

On the $\mathrm{x}$-direction, the speed of sound is

$$
c=\sqrt{\frac{\gamma p}{\rho}}
$$

the Alfvén speed is

$$
c_{a}=\frac{\left|B_{x}\right|}{\sqrt{\rho}}
$$

and the fast and slow speeds are given by

$$
c_{f, s}=\sqrt{\frac{1}{2}\left[c^{2}+b^{2} \pm \sqrt{\left(c^{2}+b^{2}\right)^{2}-4 c^{2} c_{a}^{2}}\right]}
$$

where $b^{2}=\frac{B_{x}^{2}+B_{y}^{2}+B_{z}^{2}}{\rho}$.

\subsection{E-CUSP scheme for MHD equations}

For hydrodynamics, the characteristic analysis is given as the foundation to construct the E-CUSP [32,33,35,36]. The basic idea is to split the flux $\mathbf{E}$ to the convective flux $\mathbf{F}^{\mathbf{c}}$ and the pressure flux $\mathbf{F}^{\mathbf{p}}$. That is

$$
\mathbf{E}=\mathbf{F}^{\mathbf{c}}+\mathbf{F}^{\mathbf{p}}=\left(\begin{array}{l}
\rho u \\
\rho u^{2} \\
\rho u v \\
\rho u w \\
\rho e u
\end{array}\right)+\left(\begin{array}{l}
0 \\
p \\
0 \\
0 \\
p u
\end{array}\right) .
$$

The vector $\mathbf{F}^{\mathbf{c}}$ has the eigenvalues of the velocity $(u, u, u, u, u)$, which represent the convective terms. The vector $\mathbf{F}^{\mathbf{p}}$ has the eigenvalues of the speed of sound, $(0,0,0,-a, a)$, which represents the acoustic waves propagating in each direction at subsonic regime. Based on the above separation of convective and wave terms in subsonic regime, Zha et al. [32,33,35,36] suggested to treat the convective term $\mathbf{F}^{\mathbf{c}}$ in an upwind manner and to average the wave term $\mathbf{F}^{\mathbf{p}}$ in both upwind and downwind direction with the weight of $u \pm a$.

For magnetohydrodynamics eigensystem, the eigenvalues contain three different wave speeds, i.e., the fast wave speed $c_{f}$, the slow wave speed $c_{s}$ and the Alfvén wave speed $c_{a}$. Due to its complexity, so far there are no exact formulations for the wave fluxes corresponding to the $\mathbf{F}^{\mathbf{p}}$ in hydrodynamics. However, since the ideal MHD equations have a wave-like structure analogous to that of hydrodynamics, the basic idea of E-CUSP can be extended to solve the MHD equations.

The semi-discretized conservative one-dimensional MHD equations can be written as

$$
\frac{d \mathbf{U}}{d t}+\frac{1}{\Delta x}\left(\mathbf{E}_{i+1 / 2}-\mathbf{E}_{i-1 / 2}\right)=0 .
$$

Following the E-CUSP scheme of Zha et al. [36], the flux E may be decomposed to convective and generalized wave fluxes as the following,

$$
\mathbf{E}=\mathbf{f} u+\mathbf{P}+\psi u,
$$


where

$$
\mathbf{f}=\left\{\begin{array}{l}
\rho \\
\rho u \\
\rho v \\
\rho w \\
\rho e \\
B_{x} \\
B_{y} \\
B_{z}
\end{array}\right\}, \quad \mathbf{P}=\left\{\begin{array}{l}
0 \\
p_{t}-B_{x} B_{x} \\
-B_{y} B_{x} \\
-B_{z} B_{x} \\
-B_{x}\left(u B_{x}+v B_{y}+w B_{z}\right) \\
-u B_{x} \\
-v B_{x} \\
-w B_{x}
\end{array}\right\}, \quad \psi=\left\{\begin{array}{l}
0 \\
0 \\
0 \\
0 \\
p_{t} \\
0 \\
0 \\
0
\end{array}\right\} .
$$

Similar to the pressure term pu that is separated from the enthalpy term $\rho \mathbf{H} u$ in the E-CUSP scheme, the term $p_{t} u(\psi u)$ is also separated.

The numerical flux of the E-CUSP scheme is constructed based on the one given in [36] as the following,

$$
\mathbf{E}_{1 / 2}=a_{1 / 2}\left[C^{+} \mathbf{f}_{L}+C^{-} \mathbf{f}_{R}\right]+\left[D_{L}^{+} \mathbf{P}_{L}+D_{R}^{-} \mathbf{P}_{R}\right]+\psi_{1 / 2},
$$

where

$$
\begin{aligned}
& M_{L, R}=\frac{u_{L, R}}{a_{1 / 2}}, \\
& C^{+}=\alpha_{L}^{+}\left(1+\beta_{L}\right) M_{L}-\frac{1}{4} \beta_{L}\left(M_{L}+1\right)^{2}, \\
& C^{-}=\alpha_{R}^{-}\left(1+\beta_{R}\right) M_{R}+\frac{1}{4} \beta_{R}\left(M_{R}-1\right)^{2}, \\
& \alpha_{L, R}^{ \pm}=\frac{1}{2}\left[1 \pm \operatorname{sign}\left(M_{L, R}\right)\right], \\
& \beta_{L, R}=-\max \left[0,1-\operatorname{int}\left(\left|M_{L, R}\right|\right)\right], \\
& D_{L, R}^{ \pm}=\alpha_{L, R}^{ \pm}\left(1+\beta_{L, R}\right)-\frac{1}{2} \beta_{L, R}\left(1 \pm M_{L, R}\right)
\end{aligned}
$$

and

$$
\psi_{1 / 2}=a_{1 / 2}\left(C^{+}+C^{-}\right)\left(D^{+} \psi_{L}+D^{-} \psi_{R}\right) .
$$

Note that, in [37], the speed of a fast magnetosonic wave is used to define the Mach number $M=\frac{u}{c_{f}}$, which means $M_{L, R}$ is defined as $M_{L, R}=\frac{u_{L, R}}{C_{f_{1 / 2}}}$. However, the generalized wave fluxes (or the generalized pressure terms P) in Eq. (6) are no longer dominated by one single wave and how to estimate the relative Mach number $M_{L, R}$ is still an open question. As indicated by Shang [40], the fast wave has the same propagation speed as the speed of sound at low and high frequency, but is greater than the speed of sound at the mid-range frequency. In the present study, we find that using $M_{L, R}=\frac{u_{L, R}}{\left(C_{f}+C\right)_{1 / 2}}$ the results are more robust due to averaging the fastest two wave propagation speeds. Hence, the relative Mach number is defined as

$$
M_{L, R}=\frac{u_{L, R}}{a_{1 / 2}}
$$

where

$$
a_{1 / 2}=\frac{1}{2}\left(C_{f_{L}}+C_{L}+C_{f_{R}}+C_{R}\right) \quad \text { is adopted. }
$$

\subsection{Constrained transport method [41]}

For numerical simulation of the magnetohydrodynamic (MHD) equations, a crucial issue is to preserve the divergencefree condition $\nabla \cdot \mathbf{B}=0$ for the magnetic field $\mathbf{B}$. There are several approaches to deal with this problem. Powell et al. [3] added a source term that is proportional to $\nabla \cdot \mathbf{B}$ to the original set of MHD equations, and present a set of characteristic system. However, this system may generate some uncertainty [42]. The projection method proposed in [43] has been widely used. The projection involves the solution of a Poisson equation and also restricts the choice of boundary conditions. The constrained transport (CT) method by Evans and Hawley [44] is another approach to keep $\nabla \cdot \mathbf{B}$ to the accuracy of machine round-off error. Toth [45] found the flux constrained transport method was one of the most accurate schemes that he tested. This approach has been combined with various shock-capturing schemes by different researchers [6,46,47,41,48-52].

In this paper, the constrained transport method proposed by Balsara and Spicer [41] is adopted. For completeness, the important formulas are given as following. First, in Eq. (1), Faraday's equation can be written as

$$
\frac{\partial \mathbf{B}}{\partial t}+\nabla \times \mathbf{E}=0 .
$$


For ideal MHD the electric field $\mathbf{E}$ is given by

$$
\mathbf{E}=-\mathbf{v} \times \mathbf{B} .
$$

In the constrained transport method, the magnetic field $\mathbf{B}$ in Eq. (11) is to be treated as an area-weighted average $\overline{\mathbf{B}}$ on the zone face [41].The electric fields $\mathbf{E}$ are collocated at zone edges. Then the line integral of the electric field over a zone edge gives the electromotive force over that edge. Hence, the semi-discrete form of Eq. (11) is given by

$$
\begin{aligned}
\frac{d}{d t} \bar{B}_{x_{i-1 / 2, j, k}} & =-\frac{E_{z i-1 / 2, j+1 / 2, k}-E_{z i-1 / 2, j-1 / 2, k}}{\delta y}+\frac{E_{y_{i-1 / 2, j, k+1 / 2}}-E_{y_{i-1 / 2, j, k-1 / 2}}}{\delta z} \\
\frac{d}{d t} \bar{B}_{y_{i, j-1 / 2, k}} & =\frac{E_{z i+1 / 2, j-1 / 2, k}-E_{z i-1 / 2, j-1 / 2, k}}{\delta x}-\frac{E_{x i, j-1 / 2, k+1 / 2}-E_{x i, j-1 / 2, k-1 / 2}}{\delta z} \\
\frac{d}{d t} \bar{B}_{z_{i, j, k-1 / 2}} & =-\frac{E_{y_{i+1 / 2, j, k-1 / 2}}-E_{y_{i-1 / 2, j, k-1 / 2}}}{\delta x}+\frac{E_{x i, j+1 / 2, k-1 / 2}-E_{x i, j-1 / 2, k-1 / 2}}{\delta y}
\end{aligned}
$$

where

$$
\begin{aligned}
& E_{x i, j+1 / 2, k+1 / 2}=\frac{1}{4}\left(G_{7 i, j, k+1 / 2}+G_{7 i, j+1, k+1 / 2}-F_{8 i, j+1 / 2, k}-F_{8 i, j+1 / 2, k+1}\right), \\
& E_{y_{i+1 / 2, j, k+1 / 2}}=\frac{1}{4}\left(E_{8 i+1 / 2, j, k}+E_{8 i+1 / 2, j, k+1}-G_{6 i, j, k+1 / 2}-G_{6 i+1, j, k+1 / 2}\right), \\
& E_{z i+1 / 2, j+1 / 2, k}=\frac{1}{4}\left(F_{6 i, j+1 / 2, k}+F_{6 i+1, j+1 / 2, k}-E_{7 i+1 / 2, j, k}-E_{7 i+1 / 2, j+1, k}\right),
\end{aligned}
$$

where $E_{n}, F_{n}, G_{n}(n=6,7,8)$ is the $n$th flux of the E-CUSP scheme from above subsection.

For $2 \mathrm{D}$ case, Eq. (14) reduce to

$$
\begin{aligned}
& E_{x i, j+1 / 2}=-F_{8 i, j+1 / 2}, \\
& E_{y i+1 / 2, j}=E_{8 i+1 / 2, j}, \\
& E_{z i+1 / 2, j+1 / 2}=\frac{1}{4}\left(F_{6 i, j+1 / 2}+F_{6 i+1, j+1 / 2}-E_{7 i+1 / 2, j}-E_{7 i+1 / 2, j+1}\right) .
\end{aligned}
$$

The magnetic fields stored on the faces $\overline{\mathbf{B}}$ are averaged to the zone center value $\mathbf{B}$. They are then used to correct the energy density for the new magnetic field [41],

$$
\rho e=\rho e+\frac{1}{2}\left(\left(\overline{\mathbf{B}}^{n+1}\right)^{2}-\left(\mathbf{B}^{n+1}\right)^{2}\right)
$$

\subsection{High order WENO reconstruction [53]}

The WENO scheme is used to evaluate the conservative variables $U^{L}$ and $U^{R}$. The WENO scheme for a variable $u^{L}$ can be written as:

$$
u_{i+1 / 2}^{L}=\sum_{k=0}^{r} \omega_{k} q_{k}
$$

where $\omega_{k}(k=0, \ldots, r)$ are the weights, and the $q_{k}(k=0, \ldots, r)$ are the $r$ th order accuracy reconstruction of the variables in three different stencils.

$$
\omega_{k}=\frac{\alpha_{k}}{\alpha_{0}+\cdots+\alpha_{r-1}}
$$

where

$$
\alpha_{k}=\frac{C_{k}}{\left(\varepsilon+I S_{k}\right)^{p}}, \quad k=0,1,2
$$

and where $C_{k}$ are the optimal weights with the values given below.

The smoothness indicators $I S_{k}$ suggested by Jiang and Shu [53] are given by

$$
I S_{k}=\sum_{l=1}^{r-1} \Delta x^{2 l-1} \int_{x_{i-\frac{1}{2}}}^{x_{i+\frac{1}{2}}}\left(\frac{d^{l}}{d x^{l}} \hat{q}_{k}(x)\right)^{2} d x .
$$

The $\varepsilon$ in Eq. (19) is introduced to avoid the denominator becoming zero. Jiang and Shu's numerical tests indicate that the results are not sensitive to the choice of $\varepsilon$ as long as it is in the range of $10^{-5}-10^{-7}$. In their paper [53], $\varepsilon$ is taken as $10^{-6}$. In [54], Shen et al. suggested to use an optimized $\varepsilon$ value of $10^{-2}$ in the smoothness estimators to achieve optimal weight in smooth regions in order to minimize dissipation and improve convergence. 
The $u^{R}$ is constructed symmetrically as $u^{L}$ about $i+1 / 2$.

For the third-order $(r=2)$ WENO scheme, there are

$$
q_{0}=-\frac{1}{2} u_{i-1}+\frac{3}{2} u_{i}, \quad q_{1}=\frac{1}{2} u_{i}+\frac{1}{2} u_{i+1}
$$

and

$$
\begin{aligned}
& C_{0}=1 / 3, \quad C_{1}=2 / 3, \\
& I S_{0}=\left(u_{i}-u_{i-1}\right)^{2}, \quad I S_{1}=\left(u_{i+1}-u_{i}\right)^{2} .
\end{aligned}
$$

For the fifth-order $(r=3)$ WENO scheme, there are

$$
\left\{\begin{array}{l}
q_{0}=\frac{1}{3} u_{i-2}-\frac{7}{6} u_{i-1}+\frac{11}{6} u_{i}, \\
q_{1}=-\frac{1}{6} u_{i-1}+\frac{5}{6} u_{i}+\frac{1}{3} u_{i+1}, \\
q_{2}=\frac{1}{3} u_{i}+\frac{5}{6} u_{i+1}-\frac{1}{6} u_{i+2},
\end{array}\right.
$$

and

$$
C_{0}=0.1, \quad C_{1}=0.6, \quad C_{2}=0.3 .
$$

The $I S_{k}$ are

$$
\left\{\begin{array}{l}
I S_{0}=\frac{13}{12}\left(u_{i-2}-2 u_{i-1}+u_{i}\right)^{2}+\frac{1}{4}\left(u_{i-2}-4 u_{i-1}+3 u_{i}\right)^{2} \\
I S_{1}=\frac{13}{12}\left(u_{i-1}-2 u_{i}+u_{i+1}\right)^{2}+\frac{1}{4}\left(u_{i-1}-u_{i+1}\right)^{2} \\
I S_{2}=\frac{13}{12}\left(u_{i}-2 u_{i+1}+u_{i+2}\right)^{2}+\frac{1}{4}\left(3 u_{i}-4 u_{i+1}+u_{i+2}\right)^{2} .
\end{array}\right.
$$

\subsection{Time marching Runge-Kutta method}

The 3rd-order TVD Runge-Kutta method developed by Shu and Osher [55] is used in this paper. To solve the equation

$$
\frac{d u}{d t}=L(u),
$$

the 3rd-order TVD Runge-Kutta method is

$$
\left\{\begin{array}{l}
u^{(1)}=u^{(0)}+\Delta t L\left(u^{(0)}\right), \\
u^{(2)}=\frac{3}{4} u^{(0)}+\frac{1}{4} u^{(1)}+\frac{1}{4} \Delta t L\left(u^{(1)}\right), \\
u^{(3)}=\frac{1}{3} u^{(0)}+\frac{2}{3} u^{(2)}+\frac{2}{3} \Delta t L\left(u^{(2)}\right) .
\end{array}\right.
$$

\section{Numerical examples}

\subsection{One-dimensional Riemann problems}

(1) Brio-Wu shock tube problem.

The initial left and right values have been suggested by Brio and Wu [1] and are commonly used to test numerical schemes for one-dimensional ideal MHD. Note that the hydrodynamics data used here are identical to those in Sod's shock tube Riemann problem.

$$
\left(\rho, u, v, w, B_{y}, B_{z}, p\right)=\left\{\begin{array}{l}
(1.0,0,0,0,+1,0,1.0), \quad \text { for } x<0 \\
(0.125,0,0,0,-1,0,0.1), \text { for } x>0
\end{array}\right.
$$

with $B_{x}=0.75, \gamma=2$.

The numerical example involves a compound wave, which is a typical feature of the solutions of MHD systems. For each quantity, the solution contains five constant states separated by a fast rarefaction wave, a slow compound wave, a slow shock, and a fast rarefaction. The density presents a sixth constant state because this variable is discontinuous across the contact discontinuity [1].

Fig. 1 shows the solution with 800 points at $t=0.2$. It can be seen that the present method resolves well all the complex waves.

(2) High Mach number shock tube problem

In the second tested case the following initial values are used to demonstrate the robustness of the present scheme for high Mach number flow in MHD. The Mach number corresponding to the right-moving shock wave is 15.5. This problem is also used in $[1,4]$. 


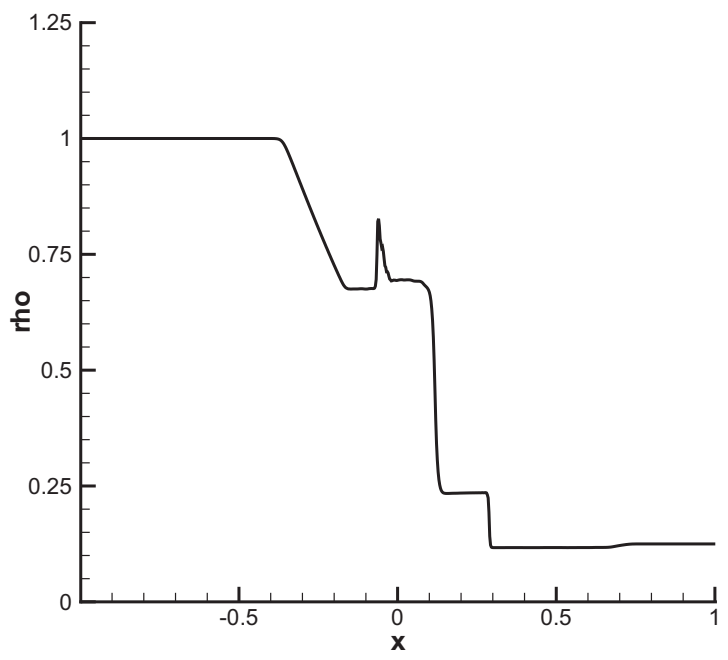

a. Density

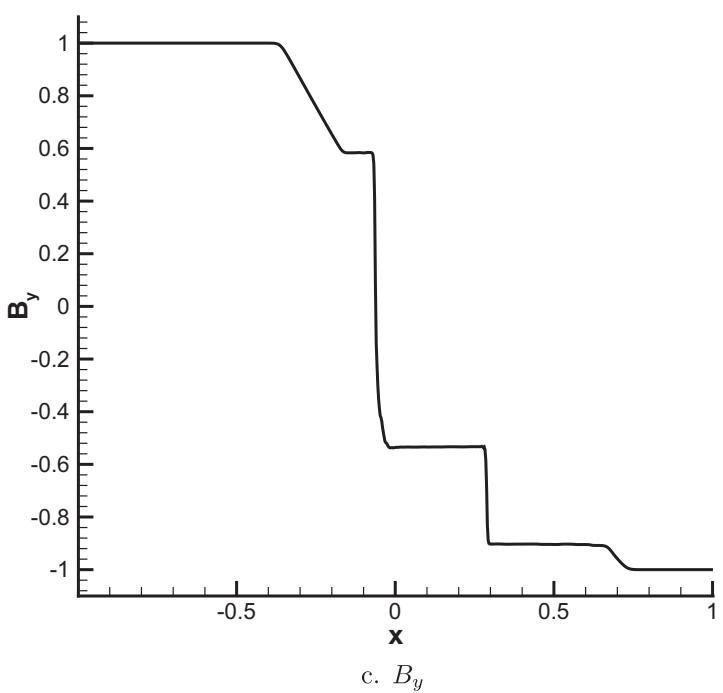

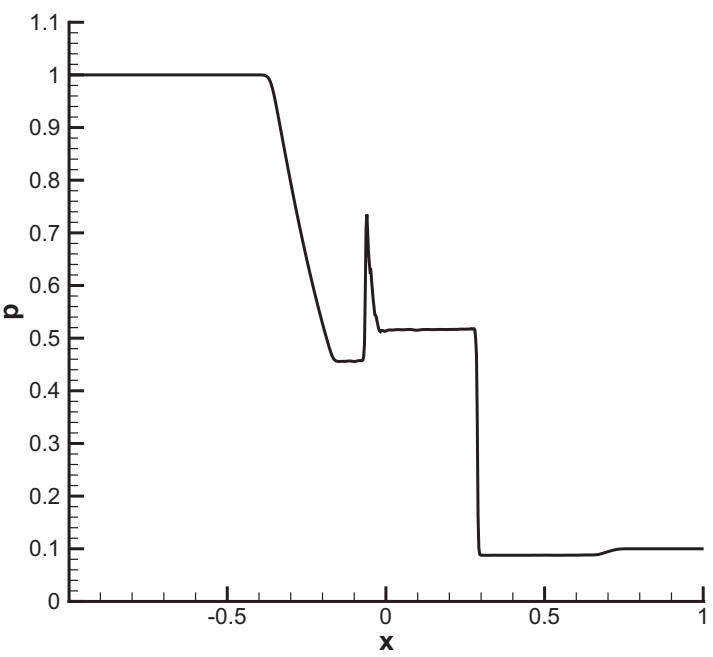

b. Pressure



d. Velocity, u

Fig. 1. Brio-Wu shock tube problem.

$$
\left(\rho, u, v, w, B_{y}, B_{z}, p\right)= \begin{cases}(1.0,0,0,0,+1,0,1000), & \text { for } x<0 \\ (0.125,0,0,0,-1,0,0.1), & \text { for } x>0\end{cases}
$$

with $B_{x}=0, \gamma=2$.

The numerical result with 200 points at $t=0.012$ is shown in Fig. 2. There is a slight undershoot at the tail of the rarefaction wave. The contact discontinuity and shock wave are captured very well. These numerical results agree well with those of Jiang and $\mathrm{Wu}[4]$ and show that the present scheme can deal well with MHD high Mach number flow.

\subsection{Two-dimensional iso-density MHD vortex advection}

The 2D vortex advection problem proposed in [56] and lately considered by Dumbser et al. [57] and Mignone at al. [58] is used to test the scheme's accuracy. The computational conditions are taken as the same used in [57,58]. The uniform meshes on the box $[-5,5] \times[-5,5]$ with the following initial conditions are adopted.

$$
\begin{aligned}
& \rho=1, \\
& u=1-y k e^{q\left(1-r^{2}\right)}, \quad v=1+x k e^{q\left(1-r^{2}\right)}, \\
& B_{x}=-y \mu e^{q\left(1-r^{2}\right)}, \quad B_{y}=x \mu e^{q\left(1-r^{2}\right)}, \\
& p=1+\frac{1}{4 q}\left(\mu^{2}\left(1-2 q r^{2}\right)-k^{2} \rho\right) e^{2 q\left(1-r^{2}\right)}
\end{aligned}
$$




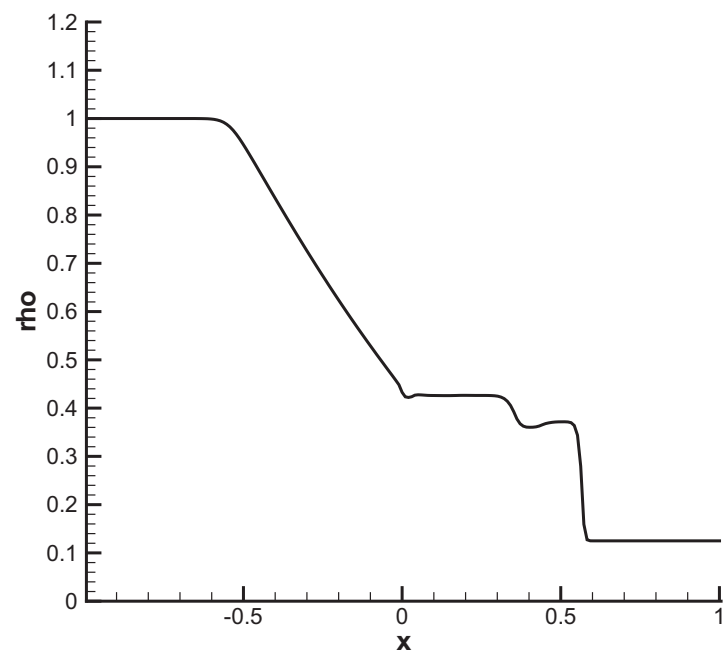

a. Density

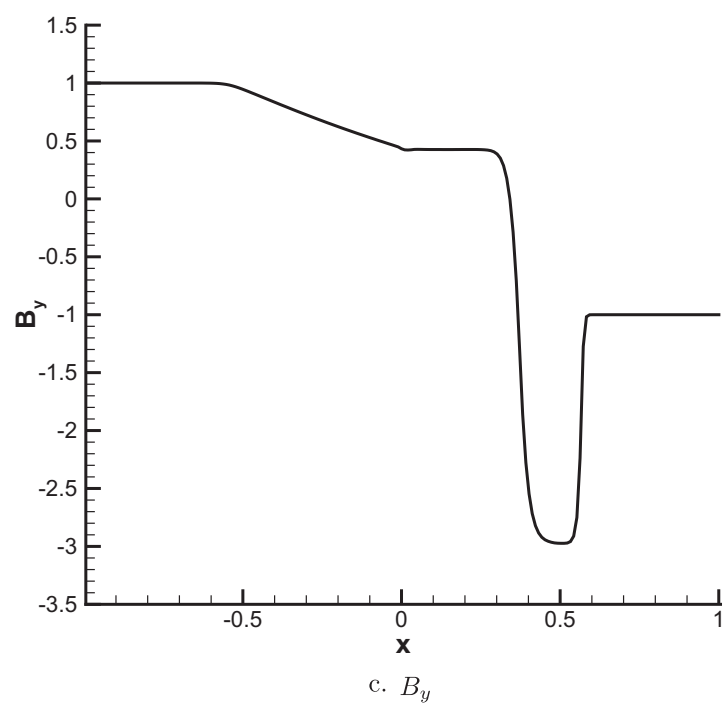



b. Pressure

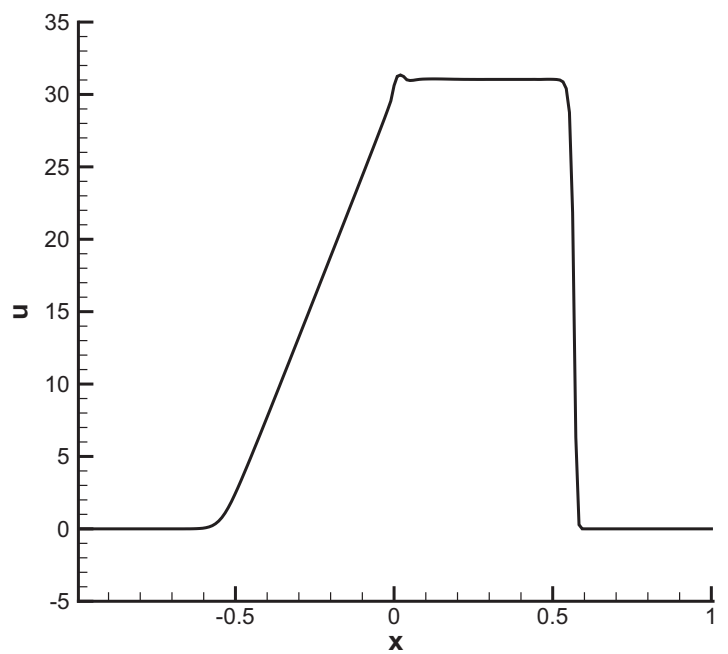

d. Velocity, u

Fig. 2. High Mach number shock tube problem.

where, $k=\mu=\frac{1}{2 \pi}, r^{2}=x^{2}+y^{2}$. The parameter $q=0.5$ and $q=1$ are chosen for the third- and fifth-order WENO reconstruction, respectively. The results are calculated at final time $t=10$ with periodic boundary conditions. The exact solution is given by Eq. (25). Since the solution is smooth, the CT method is not used in this case.

Table 1 shows the errors for $B_{x}$ measured both in $L_{\infty}$ and $L_{1}$ norms and the corresponding convergence rates. The scheme can almost reach third- and fifth-order accuracy by using the third- and fifth-order WENO reconstruction, respectively. As analyzed by Dumbser et al. [57], $B_{x}$ and $B_{y}$ have opposite signs on the left and right and on the top and bottom boundary, respectively. Hence, there is a jump in the magnetic field at the boundaries due to the periodic boundary conditions. These discontinuities cause first-order disturbances of order $10^{-5}$. For high order accurate schemes on sufficiently fine meshes, the small jump at the boundary will lead to dominant first-order errors.

\subsection{Two-dimensional circularly polarized Alfven waves}

The 2D circularly polarized Alfven wave problem is used as the second example to test the accuracy of the schemes for smooth flow. The computational conditions are same as in Ref. [45]. The circularly polarized Alfvén wave propagates at an angle $\alpha=30^{\circ}$ relative to the $x$-axis. The computational region is periodic with $0<x<1 / \cos (\alpha)$ and $0<y<1 / \sin (\alpha)$. At $t=0$, 
Table 1

Accuracy for the 2D MHD vortex advection problem, $t=10$.

\begin{tabular}{|c|c|c|c|c|c|}
\hline Scheme & $N_{x} \times N_{y}$ & $L_{\infty}$ error & $L_{\infty}$ order & $L_{1}$ error & $L_{1}$ order \\
\hline \multirow{5}{*}{ WENO-3 } & $10 \times 10$ & 0.1021 & - & $1.4958 \mathrm{e}-2$ & - \\
\hline & $20 \times 20$ & $7.6894 \mathrm{e}-2$ & 0.409 & $9.8836 \mathrm{e}-3$ & 0.598 \\
\hline & $40 \times 40$ & $2.9000 \mathrm{e}-2$ & 1.407 & $3.1625 e-3$ & 1.644 \\
\hline & $60 \times 60$ & $1.0559 \mathrm{e}-2$ & 2.491 & $8.1753 e-4$ & 3.336 \\
\hline & $80 \times 80$ & $4.7367 e-3$ & 2.787 & $3.2955 \mathrm{e}-4$ & 3.158 \\
\hline \multirow{5}{*}{ WENO-5 } & $10 \times 10$ & $9.7568 \mathrm{e}-2$ & - & $1.4971 \mathrm{e}-2$ & - \\
\hline & $20 \times 20$ & $4.2204 \mathrm{e}-2$ & 1.209 & $5.1895 \mathrm{e}-3$ & 1.511 \\
\hline & $40 \times 40$ & $3.6914 \mathrm{e}-3$ & 3.515 & $4.2682 \mathrm{e}-4$ & 3.604 \\
\hline & $60 \times 60$ & $5.2234 \mathrm{e}-4$ & 4.823 & $4.2196 \mathrm{e}-5$ & 5.707 \\
\hline & $80 \times 80$ & $1.4478 \mathrm{e}-4$ & 4.460 & $1.1927 \mathrm{e}-5$ & 4.392 \\
\hline
\end{tabular}

$$
\begin{aligned}
& \rho=1, \quad p-0.1, \\
& v_{\|}=0, \quad B_{\|}=1, \\
& v_{\perp}=B_{\perp}=0.1 \sin [2 \pi(x \cos \alpha+y \sin \alpha)], \\
& w=B_{z}=0.1 \cos [2 \pi(x \cos \alpha+y \sin \alpha)],
\end{aligned}
$$

where $B_{\perp}=B_{y} \cos \alpha-B_{x} \sin \alpha, B_{\|}=B_{x} \cos \alpha+B_{y} \sin \alpha, \gamma=5 / 3$.

For comparison, the Lax-Friedrichs (L-F) splitting method with the high order WENO schemes is also used to calculate this problem. For example, the flux $\mathbf{E}$ is split into $\mathbf{E}^{ \pm}=\frac{1}{2}(\mathbf{E} \pm a \mathbf{U})$, where $a=\max _{x, y}\left(|u|+c_{f}\right)$ is the maximal eigenvalue. The numerical flux is $E_{i+1 / 2, j}=\left(E^{+}+E^{-}\right)_{i+1 / 2, j}$, where $E_{i+1 / 2, j}^{ \pm}$is constructed by using WENO schemes. Same as in previous case, the CT method is not used in this case.

Table 2 shows the errors for $B_{\perp}$ measured both in $L_{\infty}$ and $L_{1}$ norms at $t=5$ and the corresponding convergence rates. The L-F method and the ECUSP scheme can reach the expected accuracy. But the errors of the former are almost two times of the latter. That means the ECUSP scheme has lower diffusion than the L-F method.

\subsection{Two-dimensional Kelvin-Helmholtz instability}

The Kelvin-Helmholtz instability is considered as an important mechanism for momentum transfer at Earth's magnetopause boundary, which separates the solar wind flow from the Earth's magnetosphere [59,4]. In order to compare the results, the computational conditions are taken as the same used in $[4,19]$. The initial stationary configuration of the periodic model is given by

$$
\begin{aligned}
& \rho_{0}=1, \quad u=\frac{u_{0}}{2} \tanh (y / a), \quad v=w=0, \\
& p_{0}=0.5, \quad B_{x 0}=B_{y 0}=0, \quad B_{z 0}=1,
\end{aligned}
$$

\begin{tabular}{|c|c|c|c|c|c|}
\hline Scheme & $N_{x} \times N_{y}$ & $L_{\infty}$ error & $L_{\infty}$ order & $L_{1}$ error & $L_{1}$ order \\
\hline L-F & $8 \times 8$ & $8.5375 e-2$ & - & $5.2613 e-2$ & - \\
\hline \multirow[t]{3}{*}{-WENO-3 } & $16 \times 16$ & $2.2648 \mathrm{e}-2$ & 1.914 & $1.4283 \mathrm{e}-2$ & 1.881 \\
\hline & $32 \times 32$ & $3.1200 \mathrm{e}-3$ & 2.860 & $1.9105 \mathrm{e}-3$ & 2.902 \\
\hline & $64 \times 64$ & $3.8459 \mathrm{e}-4$ & 3.020 & $2.3927 \mathrm{e}-4$ & 2.997 \\
\hline L-F & $8 \times 8$ & $3.0060 \mathrm{e}-2$ & - & $1.9944 \mathrm{e}-2$ & - \\
\hline \multirow[t]{3}{*}{-WENO-5 } & $16 \times 16$ & $8.9345 \mathrm{e}-4$ & 5.072 & $6.3736 \mathrm{e}-4$ & 4.968 \\
\hline & $32 \times 32$ & $2.3784 \mathrm{e}-5$ & 5.231 & $1.6755 e-5$ & 5.249 \\
\hline & $64 \times 64$ & $7.6216 e-7$ & 4.964 & $4.9905 \mathrm{e}-7$ & 5.069 \\
\hline ECUSP & $8 \times 8$ & $6.6484 \mathrm{e}-2$ & - & $4.3874 \mathrm{e}-2$ & - \\
\hline \multirow[t]{3}{*}{-WENO-3 } & $16 \times 16$ & $1.2335 \mathrm{e}-2$ & 2.430 & $7.6532 \mathrm{e}-3$ & 2.519 \\
\hline & $32 \times 32$ & $1.6152 \mathrm{e}-3$ & 2.933 & $9.3011 \mathrm{e}-4$ & 3.041 \\
\hline & $64 \times 64$ & $1.9477 \mathrm{e}-4$ & 3.052 & $1.1565 \mathrm{e}-4$ & 3.008 \\
\hline ECUSP & $8 \times 8$ & $1.8800 \mathrm{e}-2$ & - & $1.2734 \mathrm{e}-2$ & - \\
\hline \multirow[t]{3}{*}{-WENO-5 } & $16 \times 16$ & $4.5956 \mathrm{e}-4$ & 5.354 & $3.3229 \mathrm{e}-4$ & 5.260 \\
\hline & $32 \times 32$ & $1.2130 \mathrm{e}-5$ & 5.244 & $8.2940 \mathrm{e}-6$ & 5.324 \\
\hline & $64 \times 64$ & $4.0176 \mathrm{e}-7$ & 4.916 & $2.5307 e-7$ & 5.034 \\
\hline
\end{tabular}

where $a$ denotes the width of the velocity shear layer. At $t=0$, a small perturbation of the following form is introduced,

Table 2

Accuracy for the 2D circularly polarized Alfvén wave problem, $t=5$. 


$$
\tilde{u}_{0}= \begin{cases}-\bar{u}_{0} \sin (2 \pi x / \lambda) /\left(1+y^{2}\right), & \text { if }-\frac{\lambda}{2}<x<\frac{\lambda}{2} \\ 0, & \text { otherwise. }\end{cases}
$$

The computational domain is $\left[-\frac{L}{2}, \frac{L}{2}\right] \times[0, H] . u_{0}=2, \bar{u}_{0}=0.008, L=\lambda=5 \pi, H=1, a=1$, and $\gamma=2$ are used. The periodic boundary condition is used in the $x$-direction. The free outflow condition is applied at the top boundary at $y=H$. At the low boundary of y-direction, $\rho, p$ and $B_{z}$ are symmetric and $u$ and $v$ are antisymmetric under the transformation $x \rightarrow-x$.

A Roberts transformation $[4,19]$

$$
y=\frac{H \sinh (\tau \eta / 2 H)}{\sinh (\tau / 2)}
$$

with $\tau=6$ is used to refine the grid near $y=0$. The mesh has $96 \times 60$ grid points. Fig. 3 shows the current computational results. In this calculation, the components of $B_{x}, B_{y}$, and $w$ are always set to be zero and the evolution of $B_{z}$ follows closely with the density.

\subsection{Orszag-Tang MHD turbulence problem}

Since the Orszag-Tang MHD turbulence problem [60] has many significant characteristics of MHD turbulence, such as interactions of multiple shock waves generated as the vortex evolves, it is considered as one of the standard models to validate a MHD numerical method [61,4,62,19,37].

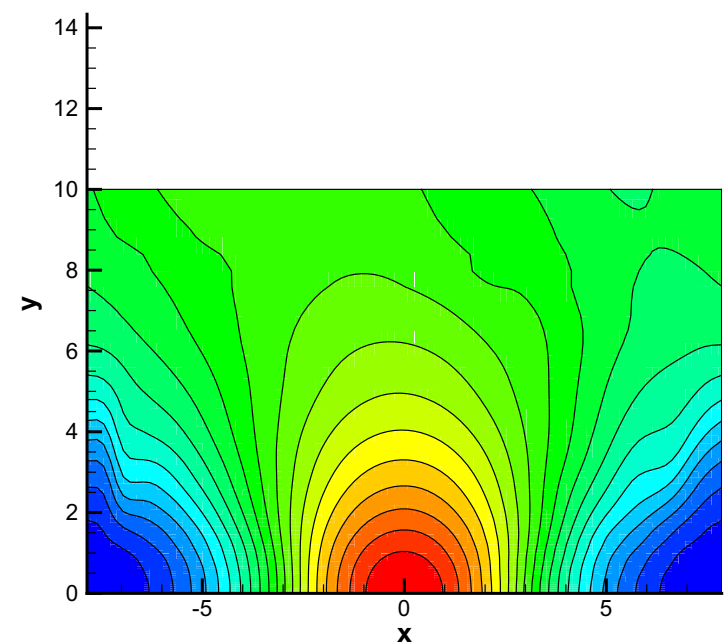

a. Density contours

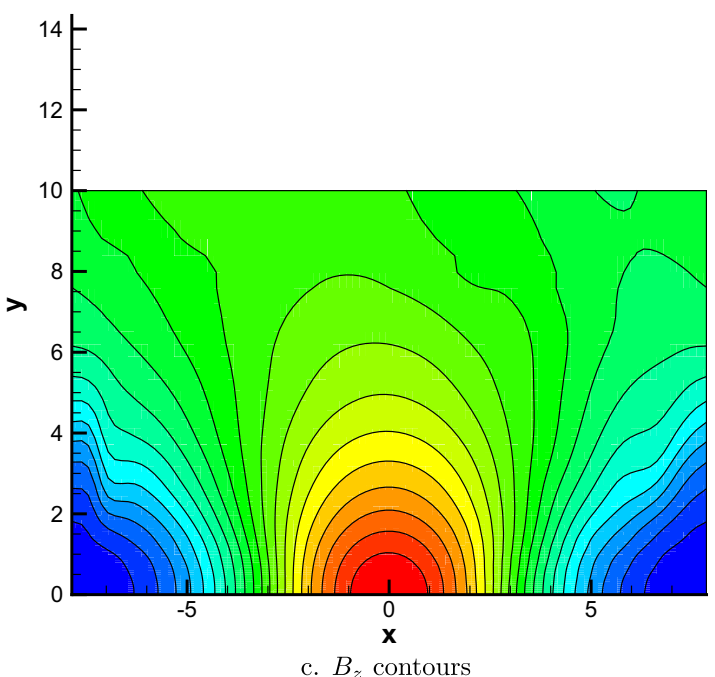



b. Pressure contours

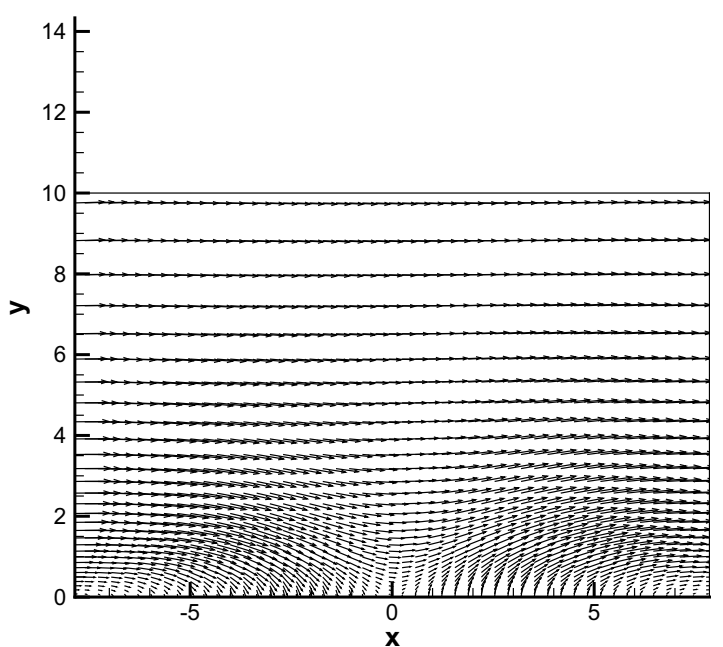

d. Velocity fields

Fig. 3. Two-dimensional Kelvin-Helmholtz instability. 
The initial conditions are given by

$$
\begin{gathered}
\rho(x, y, 0)=\gamma^{2}, \quad u(x, y, 0)=-\sin (y), \quad v(x, y, 0)=\sin (x), \\
p(x, y, 0)=\gamma, \quad B_{x}(x, y, 0)=-\sin (y), \quad B_{y}(x, y, 0)=\sin (2 x),
\end{gathered}
$$

where $\gamma=5 / 3$. As in $[61,4,62]$, the computational domain is $[0,2 \pi] \times[0,2 \pi]$. The final computation time is $t=3.0$. Periodic boundary conditions are imposed in both $x$ - and $y$-directions. Firstly, the results of the 3rd-order WENO reconstruction with and without constrained transport (CT) method are compared with a uniform mesh of $192 \times 192$ grid points. Fig. 4 shows that, without CT method, there are perturbations in both pressure field and magnetic field. With CT method, the solutions are improved greatly. Secondly, the numerical solutions with different reconstruction (the 3rd- and 5th-order WENO scheme $)$ and different meshes $(256 \times 256,512 \times 512)$ are calculated and compared. Fig. 5 displays the pressure contours, density contours, velocity field and magnetic field obtained by the 5th-order WENO reconstruction with the mesh of $256 \times 256$. In all contour figures, 20 contours are plotted. Fig. 6 shows the pressure distributions along the line of $y=1.0$ with 3rd and 5th order WENO schemes and using the different mesh sizes. These solutions agree very well in general except some minor deviation of the 3rd order scheme with the coarser mesh.

It is worthy pointing out that, if only the fast wave speed $c_{f}$ is used to calculate the relative Mach number $M_{L, R}$, i.e., $M_{L, R}=\frac{u_{L, R}}{c_{f_{1 / 2}}}$, the computation is unstable and breaks down even using third-order WENO reconstruction.

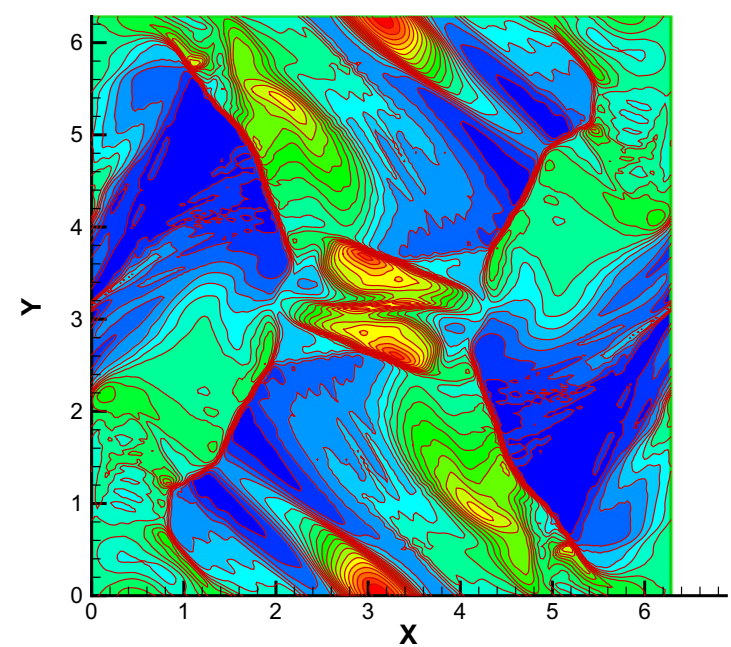

a. Pressure contours, without CT

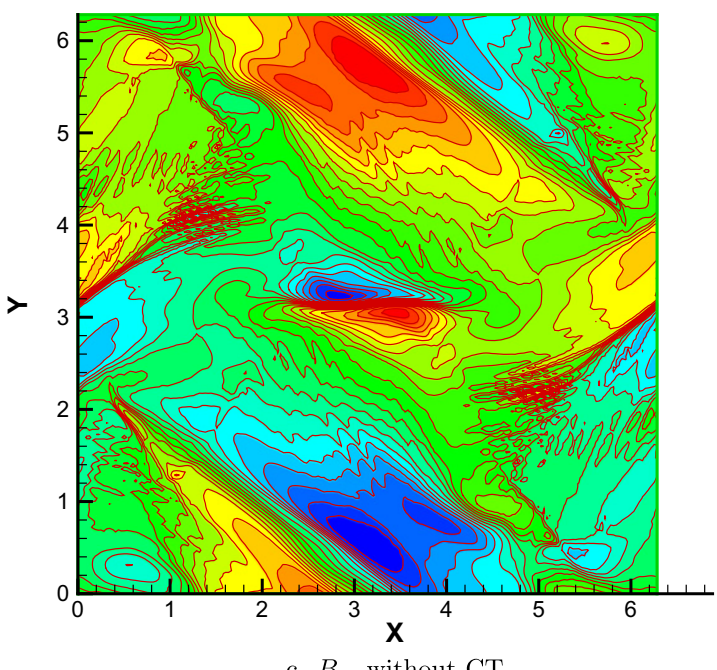

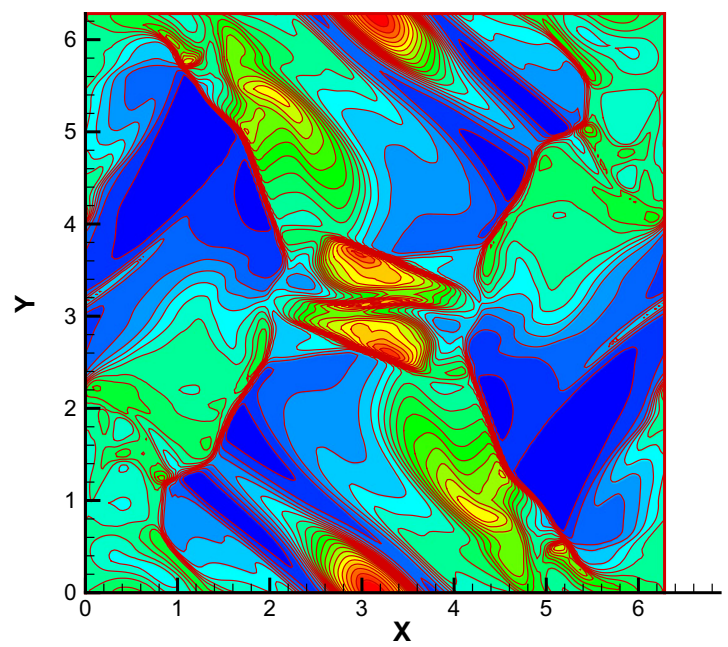

b. Pressure contours, CT

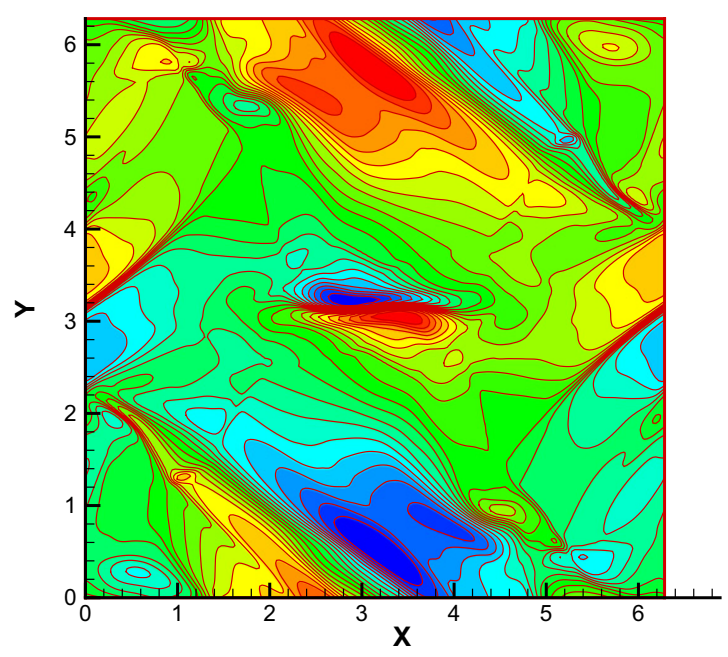

d. $B_{x}, \mathrm{CT}$

Fig. 4. Orszag-Tang MHD turbulence problem, WENO-3, $192 \times 192, t=3.0$. 


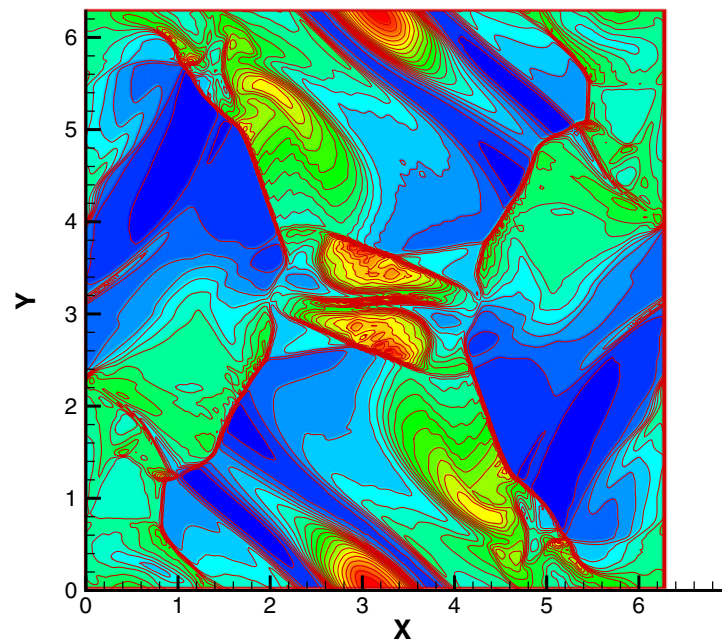

a. Pressure contours

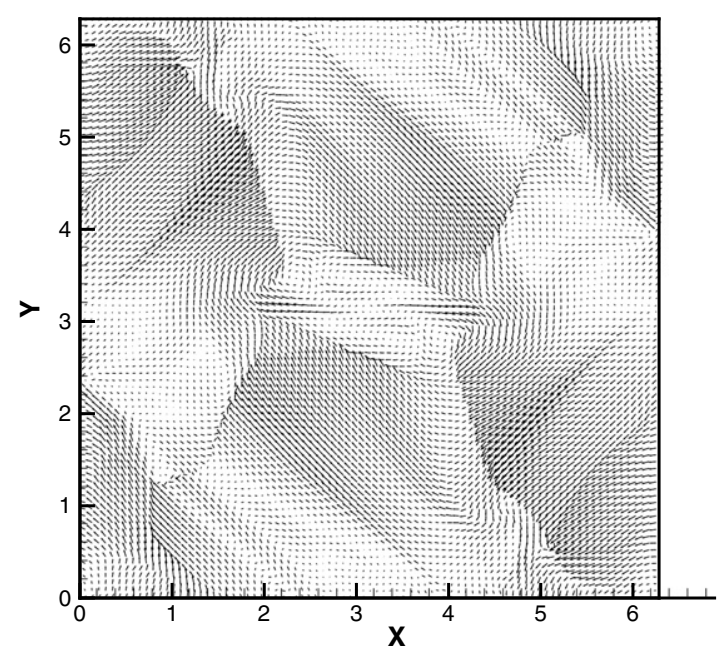

c. Velocity fields

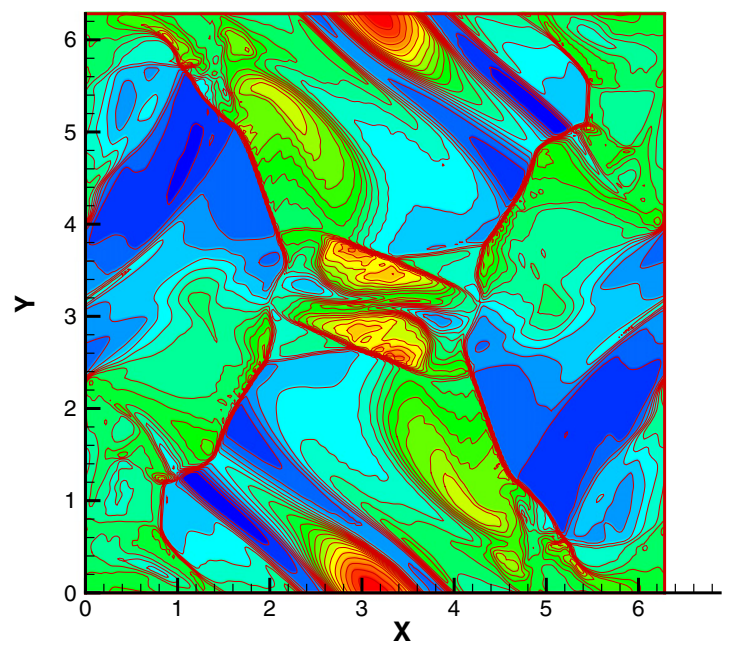

b. Density contours



d. Magnetic fields

Fig. 5. Orszag-Tang MHD turbulence problem, WENO-5, $256 \times 256, t=3.0$.



Fig. 6. Pressure distributions along the lines $y=1.0$, Orszag-Tang MHD turbulence problem, $t=3.0$. 


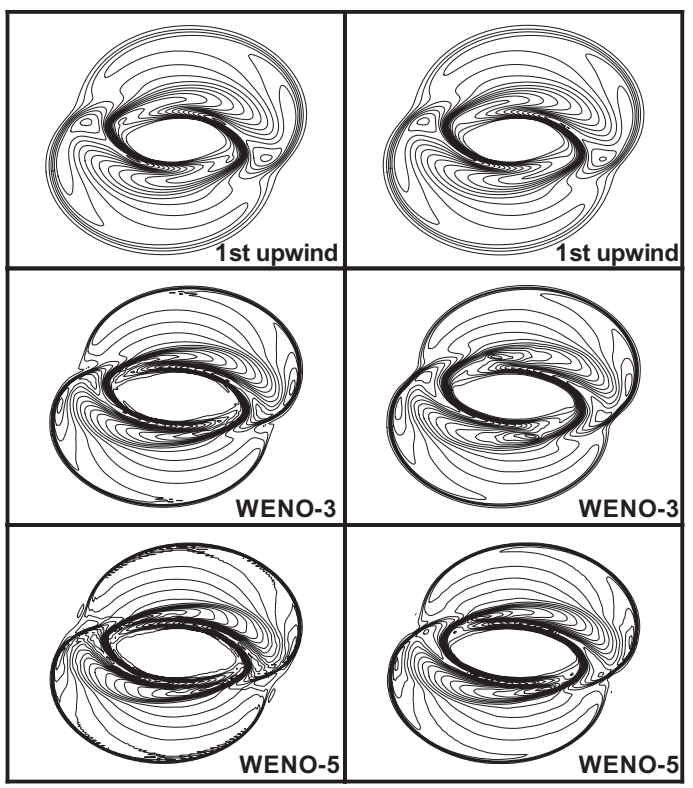

a. Pressure contours. Left: ECUSP, right: L-F. 2D rotor problem, $t=0.15$

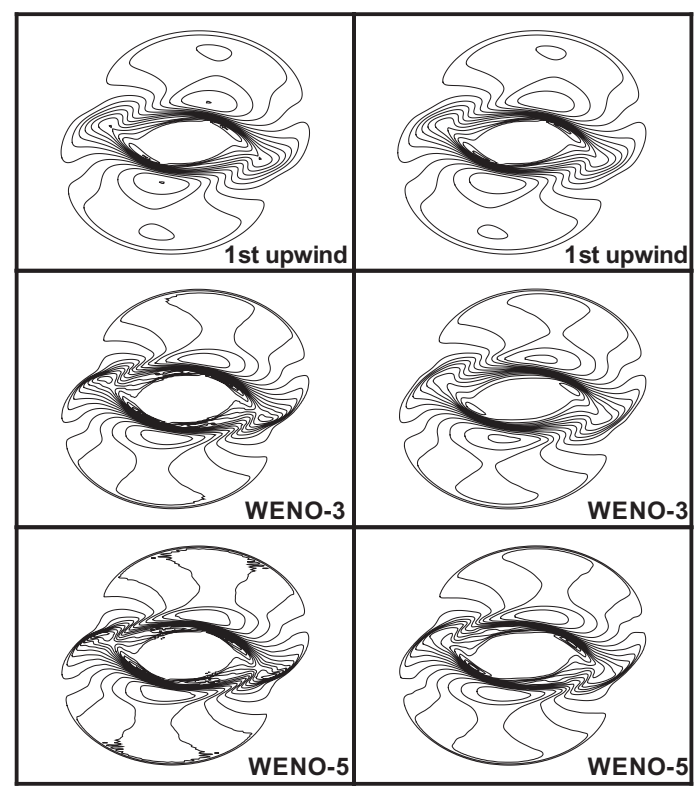

b. $B_{x}$

Fig. 7. Left: ECUSP, right: L-F. 2D rotor problem, $t=0.15$.

\section{6. $2 D \mathrm{MHD}$ rotor problem}

The rotor problem has been suggested by Balsara and Spicer [41] as a test to check the propagation of torsional Alfven waves, and then it has been widely used as a standard test model. The initial condition consists of a rapidly rotating cylinder of dense gas embedded in a lighter fluid at rest. The system is threaded by a uniform magnetic field along the $x$-axis and the problem is defined on the 2D Cartesian domain $(x, y) \in[-0.5,0.5]^{2}$

$$
\begin{aligned}
& \rho=1+9 f(r), \\
& p=1 \text {, } \\
& \left\{\begin{array}{llll}
u=-2 f(r) y / 0.1, & v=2 f(r) x / 0.1, & w=0, & \text { if } r<0.1, \\
u=-2 f(r) y / r, & v=2 f(r) x / r, & w=0, & \text { if } r \geqslant 0.1,
\end{array}\right. \\
& B_{x}=5 / \sqrt{4 \pi}, \quad B_{y}=B_{z}=0,
\end{aligned}
$$

where, $r=\sqrt{x^{2}+y^{2}}$,

$$
f(r)= \begin{cases}1 & \text { if } r<0.1 \\ \frac{200}{3}(0.115-r) & \text { if } 0.1 \leqslant r \leqslant 0.115, \\ 0 & \text { if } r>0.115\end{cases}
$$

The adiabatic index $\gamma=1.4$. For this case, when the ECUSP scheme with the third order WENO or the fifth order WENO reconstruction is used, we found the same problem as that in [63], that is: the reconstructed densities and pressures may become negative. Hence, in this paper, the first order upwind reconstruction is used to replace the reconstruction of those negative points. The L-F method has no this kind of problem. The contour plots of pressure $p$ and $x$-component of magnetic field $B_{x}$ with grid of $201 \times 201$ at the final time $t=0.15$ are shown in Fig. 7. It can be seen that the ECUSP scheme with the third order WENO reconstruction almost approaches to the L-F method with the fifth order WENO scheme. Except the negative reconstructed densities and pressures, the ECUSP scheme with the fifth order WENO reconstruction also generates slight oscillation. This may be an indication that the intrinsic numerical dissipation provided by the ECUSP scheme is not adequate for these kind of configurations. Study of this issue is currently underway and will be reported at an upcoming paper

\section{Conclusions}

An E-CUSP scheme that avoids the complex eigenstructure of the Jacobian matrices in MHD system, is developed and used with a fifth order WENO scheme to solve 1D and 2D MHD problems. A characteristic speed of sound by averaging the fast wave speed and the acoustic speed of sound is suggested to evaluate the Mach number, which yields robust and 
accurate solutions. Six standard test cases, including two one-dimensional problems, the 2D MHD vortex advection problem, the 2D Kelvin-Helmholtz instability problem, the Orszag-Tang MHD turbulence problem and the 2D MHD rotor problem, are solved to validate the accuracy and robustness of the scheme. The numerical experiments demonstrate that the scheme can resolve the complex wave characteristics in MHD very well.

\section{Acknowledgment}

This work was partially supported in part by the U. S. Air Force Office of Scientific Research under Grants FA9550-09-10105 monitored by Dr. Robert Barker.

\section{References}

[1] M. Brio, C.C. Wu, An upwind differencing scheme for the equations of ideal magnetohydrodynamics, Journal of Computational Physics 75 (1988) $400-$ 422.

[2] P.L. Roe, D.S. Balsara, Notes on the eigensystem of magnetohydrodynamics, SIAM Journal on Applied Mathematics 56 (1996) 57-67.

[3] K.G. Powell, P.L. Roe, T.J. Linde, T.I. Gombosi, D.L. De Zeeuw, A solution-adaptive upwind scheme for ideal magnetohydrodynamics, Journal of Computational Physics 154 (1999) 284-309.

[4] Guang-Shan Jiang, Cheng-chin Wu, A high-order WENO finite difference scheme for the equations of ideal magnetohydrodynamics, Journal of Computational Physics 150 (1999) 561-594.

[5] K. Murawski, Analytical and Numerical Methods for Wave Propagation in Fluid Media, World Scientific Publising Co., 2002.

[6] W.L. Dai, P.R. Woodward, An approximate Riemann solver for ideal magnetohydrodynamics, Journal of Computational Physics 111 (1994) $354-372$.

[7] A.L. Zachary, P. Colellaz, A higher-order Godunov method for the equations of ideal magnetohydrodynamics, Journal of Computational Physics 99 (1992) 341-347.

[8] P. Cargo, G. Gallice, Roe matrices for ideal MHD and systematic construction of roe matrices for systems of conservation laws, Journal of Computational Physics 136 (1997) 446-466.

[9] P. Janhunen, A positive conservative method for magnetohydrodynamics based on HLL and Roe methods, Journal of Computational Physics 160 (2000) 649-661.

[10] V. Honkkila, P. Janhunen, HLLC solver for ideal relativistic MHD, Journal of Computational Physics 223 (2007) 643-656.

[11] K.F. Gurski, An HLLC-type approximate Riemann solver for ideal magnetohydrodynamics, SIAM Journal on Scientific Computing 25 (2004) $2165-2187$.

[12] S.T. Li, An HLLC Riemann solver for magneto-hydrodynamics, Journal of Computational Physics 203 (2005) 344-357.

[13] T. Miyoshi, K. Kusano, A multi-state HLL approximate Riemann solver for ideal magnetohydrodynamics, Journal of Computational Physics 208 (2005) 315-344.

[14] Dinshaw S. Balsara, Tobias Rumpf, Michael Dumbser, Claus-Dieter Munz, Efficient high accuracy ADER-WENO schemes for hydrodynamics and divergence-free magnetohydrodynamics, Journal of Computational Physics 228 (2009) 2480-2516.

[15] R.W. MacCormack, An Upwind Conservation form Method for Ideal Magnetohydrodynamics Equations, AIAA, 1999. Paper 99-3609.

[16] R.W. MacCormack, Non-equilibrium ionized flow simulations within strong electro-magnetic fields, AIAA, 2010-0225, 2010.

[17] C.K. Lombard, J. Bardina, E. Venkatapathy, J. Oliger, Multidimensional formulation of CSCM-an upwind flux eigenvector split method for the compressible Navier-Stokes equations, AIAA paper 83-1895, in: Proceedings of the AIAA 6th Computational Fluid Dynamics Conference, 1983, p.649664.

[18] H.C. Yee, B. Sjogreen, Development of low dissipative high order filter schemes for multiscale Navier-Stokes/MHD systems, Journal of Computational Physics 225 (2007) 910-934.

[19] J. Balbas, E. Tadmor, C.-C. Wu, Non-oscillatory central schemes for one- and two-dimensional MHD equations: I, Journal of Computational Physics 201 (2004) 261-285.

[20] D.V. Gaitonde, Development of a Solver for 3-D Non-Ideal Magnetogasdynamics, AIAA, 1999. Paper 99-3610.

[21] A. Jameson, Analysis and Design of Numerical Schemes for Gas Dynamics I: Artificial Diffusion, Upwind Biasing, Limiters and Their Effect on Accuracy and Multigrid Convergence in Transonic and Hypersonic Flow, AIAA Paper, July 1993, pp. 93-3359.

[22] A. Jameson, Analysis and Design of Numerical Schemes for Gas Dynamics I: Artificial Diffusion, Upwind Biasing, Limiters and Their Effect on Accuracy and Multigrid Convergence in Transonic and Hypersonic Flow, AIAA Paper, July 1993, pp. 93-3359.

[23] A. Jameson, Analysis and design of numerical schemes for gas dynamics II: Artificial diffusion and discrete shock structure, Journal of Computational Fluid Dynamics 5 (1995) $1-38$

[24] M.-S. Liou, C.J. Steffen, A new flux splitting scheme, Journal of Computational Physics 107 (1993) 23-39.

[25] Y. Wada, M.-S. Liou, An Accurate and Robust Splitting Scheme for Shock and Contact Discontinuities, AIAA, 1994. Paper 94-0083.

[26] M.-S. Liou, Progress Towards an Improved CFD Methods: AUSM+, AIAA Paper 95-1701-CP, June 1995.

[27] M.-S. Liou, A sequel to AUSM: AUSM+ ${ }^{+}$, Journal of Computational Physics 129 (1996) 364-382.

[28] M.-S. Liou, Ten Years in the Making-AUSM-Family, AIAA, 2001. 2001-2521.

[29] D. Hänel, R. Schwane, G. Seider, On the Accuracy of Upwind Schemes for the Solution of the Navier-Stokes Equations, AIAA, 1987. paper 87-1105 CP.

[30] J.R. Edwards, A Low-Diffusion Flux-Splitting Scheme for Navier-Stokes Calculations, AIAA, 1995. Paper 95-1703-CP, June.

[31] J.R. Edwards, A low-diffusion flux-splitting scheme for Navier-Stokes calculations, Computer and Fluids 6 (1997) 635-659.

[32] G.-C. Zha, E. Bilgen, Numerical solutions of Euler equations by using a new flux vector splitting scheme, International Journal for Numerical Methods in Fluids 17 (1993) 115-144.

[33] G.-C. Zha, Numerical tests of upwind scheme performance for entropy condition, AIAA Journal 37 (1999) 1005-1007.

[34] G.-C. Zha, Comparative Study of Upwind Scheme Performance for Entropy Condition and Discontinuities, AIAA, 1999. Paper 99-CP-3348, June 28- July 1.

[35] G.-C. Zha, Z.-J. Hu, Calculation of transonic internal flows using an efficient high resolution upwind Scheme, AIAA Journal 42 (2004) 205-214. No.2.

[36] G.C. Zha, Y.Q. Shen, B.Y. Wang, An improved low diffusion E-CUSP upwind scheme, Computers and Fluids 48 (2011) 214-220.

[37] Sang-Hoon Han, Jeong-Il Lee, Kyu Hong Kim, Accurate and robust pressure weight advection upstream splitting method for magnetohydrodynamics equations, AIAA Journal 47 (2009) 970-981.

[38] R.K. Agarwal, J. Augustinus, D.W. Halt, A Comparative Study of Advection Upwind Split (AUSM) and Wave/Particle Split (WPS) Schemes for Fluid and MHD Flows, AIAA-1999-3613, 1999.

[39] H.-M. Damevin, K.A. Hoffmann, Development of a modified Runge-Kutta scheme with TVD limiters for ideal three-dimensional magnetogasdynamics, AIAA 2001-2739, 2001.

[40] J. Shang, Recent research in magneto-aerodynamics, Progress in Aerospace Sciences 37 (2001) 1-20.

[41] D.S. Balsara, D.S. Spicer, A staggered mesh algorithm using high order Godunov fluxes to ensure solenoidal magnetic fields in magnetohydrodynamics simulation, Journal of Computational Physics 149 (1999) 270-292.

[42] Y.-Q. Shen, G.-C. Zha, M.A. Huerta, E-CUSP scheme for the equations of magnetohydrodynamics, in: 49th AIAA Aerospace Sciences Meeting, AIAA Paper 2011-383, Orlando, FL, January 2011, pp. 4-7. 
[43] J.U. Brackbill, D.C. Barnes, The Effect of Nonzero backward difference $\nabla \cdot B$ on the numerical solution of the magnetohydrodynamic equations, Journal of Computational Physics 35 (1980) 426-430.

[44] C.R. Evans, J.F. Hawley, Simulation of magnetohydrodynamic flows: a constrained transport method, Astrophysics Journal 332 (1988) 659-677.

[45] G. Toth, The $\nabla \cdot B=0$ constraint in shock-capturing magnetohydrodynamics codes, Journal of Computational Physics 161 (2000) 605-652.

[46] C.R. DeVore, Flux corrected transport techniques for multidimensional compressible magnetohydrodynamics, Journal of Computational Physics 92 (1991) 142-160.

[47] D. Ryu, F. Miniati, T.W. Jones, A. Frank, A divergence-free upwinding code for multi-dimensional MHD flows, Astrophysics Journal 509 (1998) $244-255$.

[48] P. Londrillo, L. Del Zanna, On the divergence-free condition in Godunov-type schemes for ideal magnetohydrodynamics: the upwind constrained transport method, Journal of Computational Physics 195 (2004) 17-48.

[49] U. Ziegler, A central-constrained transport scheme for ideal magnetohydrodynamics, Journal of Computational Physics 196 (2004) $393-416$.

[50] T.A. Gardiner, J.M. Stone, An unsplit Godunov method for ideal MHD via constrained transport in three dimensions, Journal of Computational Physics 227 (2008) 4123-4141.

[51] Shengtai Li, A fourth-order divergence-free method for MHD flows, Journal of Computational Physics 229 (2010) $7893-7910$.

[52] C. Helzel, J.A. Rossmanith, B. Taetz, An unstaggered constrained transport method for the 3D ideal magnetohydrodynamic equations, Journal of Computational Physics 230 (2011) 3803-3829.

[53] G.-S. Jiang, C.-W. Shu, Efficient implementation of weighted ENO schemes, Journal of Computational Physics 126 (1996) $202-228$.

[54] Y.-Q. Shen, G.-C. Zha, B.-Y. Wang, Improvement of stability and accuracy of implicit WENO scheme, AIAA Journal 47 (2009) $331-344$.

[55] C.-W. Shu, O. Osher, Efficient implementation of essentially non-oscillatory shock capturing schemes, Journal of Computational Physics 77 (1988) 439471.

[56] D. Balsara, Second-order accurate schemes for magnetohydrodynamics with divergence-free reconstruction, The Astrophysical Journal Supplement Series 151 (2004) 149-184.

[57] M. Dumbser, D.S. Balsara, E.F. Toro, C.-D. Munz, A unified framework for the construction of one-step finite volume and discontinuous Galerkin schemes on unstructured meshes, Journal of Computational Physics 227 (2008) 8209-8253.

[58] A. Mignone, P. Tzeferacos, G. Bodo, High-order conservative finite difference GLM-MHD schemes for cell-centered MHD, Journal of Computational Physics 229 (2010) 5896-5920.

[59] C.C. Wu, Kelvin-Helmholtz instability at the magnetopause boundary, Journal of Geophysical Research 91 (1986) $3042-3060$.

[60] S.A. Orszag, C.M. Tang, Small-scale structure of two-dimensional magnetohydrodynamic turbulence, Journal of Fluid Mechanics 90 (1979) $129-143$.

[61] A. Zachary, A. Malagoli, P. Colella, A high-order Godunov method for multidimensional ideal magnetohydrodynamics, SIAM Journal on Science Computing 15 (1994) 263-284.

[62] H.Z. Tang, K. Xu, A high-order gas-kinetic method for multidimensional ideal magnetohydrodynamics, Journal of Computational Physics 165 (2000) 69-88.

[63] F.G. Fuchs, A.D. McMurry, S. Mishra, N.H. Risebro, K. Waagan, Approximate Riemann solvers and stable high-order finite volume schemes for multidimensional ideal MHD, Research Report No. 2009-37, CH-8092 Zurich, Switzerland, Nov. 2009. 